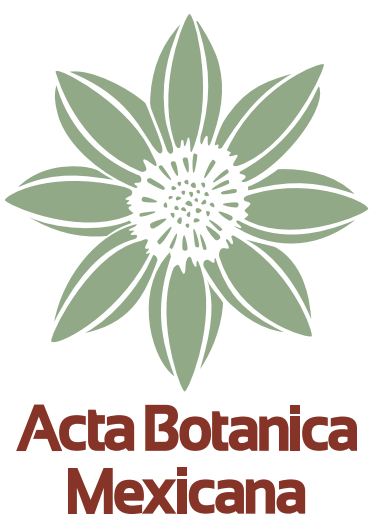

\section{Nuevo registro de hongos micorrícicos arbusculares en Nothoceros fuegiensis (Dendrocerotaceae, Anthocerotophyta)}

\author{
New record of arbuscular mycorrhizal \\ fungi in Nothoceros fuegiensis \\ (Dendrocerotaceae, Anthocerotophyta)
}

\author{
Agustina C. Cottet, ${ }^{1,2}$ (D) y María I. Messuti' (1)
}

\title{
Resumen:
}

Antecedentes y Objetivos: Los Hongos Micorrícicos Arbusculares (HMA) se encuentran asociados, en la naturaleza, con varias especies de antocerotes (Anthocerotophyta). El objetivo de este estudio es dar a conocer y caracterizar por primera vez la colonización de HMA en Nothoceros fuegiensis (Dendrocerotaceae).

Métodos: El material examinado proviene de recolectas realizadas en bosques templados fríos dominados por Nothofagus spp. Las plantas fueron acondicionadas, teñidas y analizadas de acuerdo con técnicas específicas para briofitas.

Resultados clave: Se describe por primera vez la asociación micorrícica en $N$. fuegiensis. Se presenta una descripción breve e ilustración de la planta y de las estructuras características del hongo micorrícico. El porcentaje de colonización es de 9-40\%, ocasionalmente ausente, y su tipo morfológico es Paris.

Conclusiones: Al describir la asociación entre HMA y un antocerote, $N$. fuegiensis, se aporta nueva información sobre los registros de asociaciones presentes en diferentes briofitas en ambientes poco conocidos o inexplorados.

Palabras clave: antocerotes, Argentina, asociación fúngica, Patagonia, tipo Paris.

\section{Abstract:}

Background and Aims: Arbuscular Mycorrhizal Fungi (AMF) are associated, in nature, with several species of hornworts (Anthocerotophyta). The aim of this study is to describe and characterize for the first time AMF colonization in Nothoceros fuegiensis (Dendrocerotaceae).

Methods: The material examined was collected in cold temperate forest dominated by Nothofagus spp. The plants were conditioned, stained and analyzed according to specific techniques for bryophytes.

Key results: The mycorrhizal association in $N$. fuegiensis is described for the first time. A brief description and illustration of the plant and the characteristic structures of the mycorrhizal fungus are presented. The percentage of colonization ranges from 9-40\%, occasionally absent and its morphological type is Paris.

Conclusions: The association between AMF and a hornwort, N. fuegiensis, is described, thus providing new information on the records of this type of association present in different bryophyte taxa in little-known or unexplored environments.

Key words: Argentina, fungal association, hornworts, Paris-type, Patagonia.

${ }^{1}$ Consejo Nacional de Investigaciones Científicas y Técnicas-Universidad Nacional del Comahue, Instituto de Investigaciones en Biodiversidad y Medioambiente, Quintral 1250, San Carlos de Bariloche, 8400 Río Negro, Argentina.

22Autor para la correspondencia: agustina.c.cottet@ gmail.com
Recibido: 19 de febrero de 2020

Revisado: 13 de abril de 2020.

Aceptado por Rosario Redonda-Martínez: 24 de junio de 2020.

Publicado primero en línea: 1 de julio de 2020.

Publicado: Acta Botanica Mexicana 127(2020).

(c) (i) (8) Este es un artículo de acceso abierto EY bajo la licencia Creative Commons 4.0 Atribución-No Comercial (CC BY-NC 4.0 Internacional).
Citar como: Cottet, A. C. y M. I. Messuti. 2020. Nuevo registro de hongos micorrícicos arbusculares en Nothoceros fuegiensis (Dendrocerotaceae, Anthocerotophyta). Acta Botanica Mexicana 127: e1681. DOI: 10.21829/abm127.2020.1681 


\section{Introducción}

Las asociaciones que se establecen entre Hongos Micorrícicos Arbusculares (HMA) y briofitas (Anthocerotophyta, Bryophyta y Marchantiophyta) juegan un rol importante en el desarrollo de este grupo de plantas no vasculares. Asociarse les permite establecerse en ambientes con ciertas condiciones desfavorables para su desarrollo, como pueden ser los suelos pobres en materia orgánica, temperaturas extremas y presencia de patógenos (Liepina, 2012; Nelson y Shaw, 2019). En función de las estructuras fúngicas presentes como hifas inter e intracelulares, arbúsculos, coils y coils arbusculados, estas asociaciones se clasifican en dos tipos morfológicos conocidos como Arum y Paris (Smith y Smith, 1997). Dickson (2004) propuso la existencia de formas intermedias entre ambos tipos morfológicos, reconociendo una secuencia estructural entre Arum (con hifas intercelulares y arbúsculos intracelulares) y Paris (con hifas intracelulares con coils y coils arbusculados).

La relación entre HMA y Anthocerotophyta (antocerotes) ha sido estudiada con anterioridad y se sabe que en antocerotes la colonización por hongos puede diferir en abundancia y ocurrencia en los géneros Anthoceros L., Folioceros D.C. Bhardwaj, Megaceros Campbell, Nothoceros (R.M. Schust.) J. Haseg., Notothylas Sull. ex A. Gray, Phaeoceros Prosk. y Phaeomegaceros Duff, J.C. Villarreal, Cargill \& Renzaglia, mientras que en Dendroceros Nees y Leiosporoceros Hässel, está ausente (Desirò et al., 2013). Dentro de las siete especies que comprenden el género Nothoceros (Villarreal et al., 2010), en dos ha sido analizada la presencia de asociaciones micorrícicas (Desirò et al., 2013). En Nothoceros vincentianus (Lehm. \& Lindenb.) J.C. Villarreal, se registró la presencia de HMA con base en la revisión de especímenes recolectados en Panamá (Öpik et al., 2010; Desirò et al., 2013; Feijen et al., 2018), mientras que en $N$. giganteus (Lehm. \& Lindenb.) J. Haseg. ex J.C. Villarreal, Hässel \& N. Salazar, de material proveniente de Nueva Zelanda, no se observaron asociaciones con simbiontes fúngicos (Öpik et al., 2010; Desirò et al., 2013; Feijen et al., 2018). La especie N. fuegiensis (Steph.) J.C. Villarreal no ha sido analizada, por ello el objetivo principal de este trabajo es dar a conocer y caracterizar por primera vez la colonización de HMA en este antocerote.

\section{Materiales y Métodos}

\section{Recolecta de material}

Los especímenes de $N$. fuegiensis fueron recolectados en las Provincias de Chubut (Paraje Entre Ríos, Planta Educativa Parque Nacional Lago Puelo y arroyo Centinela); Neuquén (Lagunas de Epulafquen, Pilolil-Rahue, Playa Yuco y Termas del Queñi); Río Negro (Pampa Linda, arroyo Alerce, Paso Pérez Rosales, Puerto Blest, Villa los Coihues, lago Steffen y Refugio Los Laguitos) y Santa Cruz (Puerto Argentino); en sitios de bosque templado frío dominado por especies de Nothofagus Blume en la Patagonia, Argentina. El material recolectado se acondicionó utilizando técnicas tradicionales para el estudio de plantas no vasculares (Frahm, 2003) y se depositó en el herbario BCRU (Centro Regional Universitario Bariloche, Universidad Nacional del Comahue, San Carlos de Bariloche, Argentina). Para la identificación del material examinado se utilizaron los trabajos de Hässel de Menéndez (1961, 1990) y Villarreal et al. (2010).

\section{Estudio de colonización}

Las estructuras características de los HMA (hifas aseptadas, coils, arbúsculos y vesículas) fueron observadas después de preparar secciones sagitales de gametofitos teñidos y montadas en agua o lactofenol, se observaron en un microscopio compuesto Olympus BX50 (Olympus Corp., Tokio, Japón) y se fotografiaron con una cámara digital Lumenera Infinity 1 (Lumenera Corp., Otawa, Canadá).

Para observar la colonización por HMA, los gametofitos fueron lavados con agua corriente y preservados en etanol al 70\% durante 24 horas a temperatura ambiente; posteriormente se colocaron en cajas de Petri a $50{ }^{\circ} \mathrm{C}$ sobre un agitador magnético de calentamiento (VELP Scientifica, Usmate, Velate, Italia), con placa caliente hasta la evaporación total del líquido. Posteriormente, fueron clarificados en hidróxido de potasio al $1 \%$ durante 20 minutos a $80^{\circ} \mathrm{C}$; acidificados con ácido clorhídrico al $1 \%$ durante 10 minutos a $50{ }^{\circ} \mathrm{C}$ y teñidos con azul de tripán al $0.05 \%$ durante $20 \mathrm{mi}$ nutos a $60^{\circ} \mathrm{C}$ (Cottet et al., 2018; Cottet y Messuti, 2019). El porcentaje de colonización por HMA se estimó utilizando la presencia o ausencia de las estructuras características de estos hongos con un aumento de $400 \times$ en todo el talo (47 talos). El número promedio de campos observados por talo fue de 800. El tipo de colonización se determinó utilizando 
los criterios de Dickson (2004), quien basó la clasificación en la presencia de hifas inter e intracelulares, arbúsculos, coils y coils arbusculados. Además, para determinar qué parte del talo estaba asociada con hifas, se realizaron cortes transversales tanto en las porciones distales como proximales de la planta.

\section{Resultados}

Los especímenes examinados de $N$. fuegiensis presentaron gametofitos verde-esmeralda, cespitosos o formando rosetas (Fig. 1A) adheridos al sustrato por rizoides unicelulares. En sección transversal, los talos mostraron una superficie dorsal plana con epidermis; tejido fundamental sólido sin cavernas, colonias de cianobacterias y una superficie ventral plana similar a la dorsal (Fig. 1B). En el área de estudio esta especie es frecuente, se encontró asociada a sectores muy húmedos cerca de arroyos o ríos, incluso sumergidos. Los talos analizados provenientes de sustratos no sumergidos, tales como suelo, roca y madera, presentaron una colonización de HMA variable donde el porcentaje osciló entre $9-40 \%$. En tanto que en los talos que se desarrollaban sobre suelo, roca o madera sumergidos, o anfibios, no hubo colonización. Las estructuras fúngicas características observadas fueron las siguientes: hifas superficiales o externas cercanas a los rizoides, $7 \mu \mathrm{m}$ de diámetro; hifas intracelulares, $5 \mu \mathrm{m}$ de diámetro; coils arbusculados intracelulares y abundantes en la porción media del talo, 35-45 $\mu \mathrm{m}$ de diámetro (Fig. 1C); vesículas globosas, 17-25 $\mu \mathrm{m}$ de diámetro, escasas a abundantes (Fig. 1D). La colonización se encuentra restringida a la porción media ventral del gametofito y corresponde al denominado tipo morfológico Paris.

Material examinado: ARGENTINA. Provincia Chubut, Lago Puelo, Paraje Entre Ríos, confluencia río Azul y río Quenquentreu, I.2016, A. Cottet 5552 (BCRU); Parque Nacional Lago Puelo, Planta Educativa, 30.XI.2017, A. Cottet 5556 (BCRU), 5557 (BCRU); Parque Nacional Los Alerces, arroyo Centinela, 29.I.2017, A. Cottet 5549 (BCRU). Provincia Neuquén, Las Ovejas, Lagunas de Epulafquen, laguna Superior, 08.I.2019, A. Cottet 5567 (BCRU), 5568 (BCRU); entre Pilolil y Rahue, 05.X.2018, A. Cottet 5569 (BCRU); Parque Nacional Lanín, Playa Yuco, 31.XII.2018, A. Cottet 5559 (BCRU), 5560 (BCRU), 5561 (BCRU); Termas del Queñi, 30.XII.2018, A. Cottet 5562 (BCRU), 5563 (BCRU), 5564
(BCRU), 5565 (BCRU), 5566 (BCRU). Provincia Río Negro, Parque Nacional Nahuel Huapi, Pampa Linda, 08.XII.2016, A. Cottet 5539 (BCRU); sendero Refugio Otto Meiling, arroyo Alerce, 08.XII.2016, A. Cottet 5541 (BCRU), 5542 (BCRU), 5543 (BCRU), 5544 (BCRU); Paso Pérez Rosales, 10.XII.2016, A. Cottet 5545 (BCRU), 5546 (BCRU), 5547 (BCRU), 5548 (BCRU), Puerto Blest, sendero Puerto Cántaros - Puerto Blest, 08.IX.2017, A. Cottet 5510 (BCRU), 5518 (BCRU), 5519 (BCRU), 5550 (BCRU), 5554 (BCRU), 5555 (BCRU); San Carlos de Bariloche, Villa Los Coihues, VII.2016, A. Cottet 5553 (BCRU); Parque Nacional Nahuel Huapi, lago Steffen, 15.II.2018, A. Cottet 5558 (BCRU); El Bolsón, refugio Los Laguitos, I.2017, A. Cottet 5551 (BCRU). Provincia Santa Cruz, Parque Nacional Los Glaciares, Puerto Argentino, 26.XI.2016, A. Cottet 5526 (BCRU), 5527 (BCRU), 5528 (BCRU), 5529 (BCRU), 5530 (BCRU), 5531 (BCRU), 5532 (BCRU), 5533 (BCRU), 5534 (BCRU), 5535 (BCRU), 5536 (BCRU), 5537 (BCRU), 5538 (BCRU).

\section{Discusión}

Tradicionalmente los HMA han sido incluidos en el filo Glomeromycota (Brundrett, 2004; Bidartondo y Duckett, 2010). No obstante, en la última propuesta de clasificación filogenética (Spatafora et al., 2016) los HMA fueron transferidos a nivel de subfilo junto con Mortierellomycotina y Mucoromycotina. Con base en la clasificación de Spatafora et al. (2016), entonces, los HMA se ubican tanto en Glomeromycotina como en Mucoromycotina, representantes capaces de formar asociaciones micorrícicas con plantas no vasculares. Los Glomeromycotina se caracterizan por presentar hifas intracelulares principales de 4-8 $\mu \mathrm{m}$ de diámetro, hifas de arbúsculos con un rango entre 1-3 $\mu \mathrm{m}$ y por formar vesículas. Por otro lado, los Mucoromycotina presentan hifas intracelulares principales de 3-4 $\mu \mathrm{m}$ de diámetro, hifas de arbúsculos con un rango entre 0.5-1 $\mu \mathrm{m}$ de diámetro y no forman vesículas (Field et al., 2016). Teniendo en cuenta estas diferencias morfológicas, los hongos encontrados en $N$. fuegiensis pueden incluirse dentro de Glomeromycotina. Los especímenes estudiados presentan todas las características típicas del tipo morfológico de colonización Paris, lo que coincide con lo mencionado por Smith y Smith (1997), quienes concluyeron que este tipo predomina en las briofitas. No obstante, como una excep- 


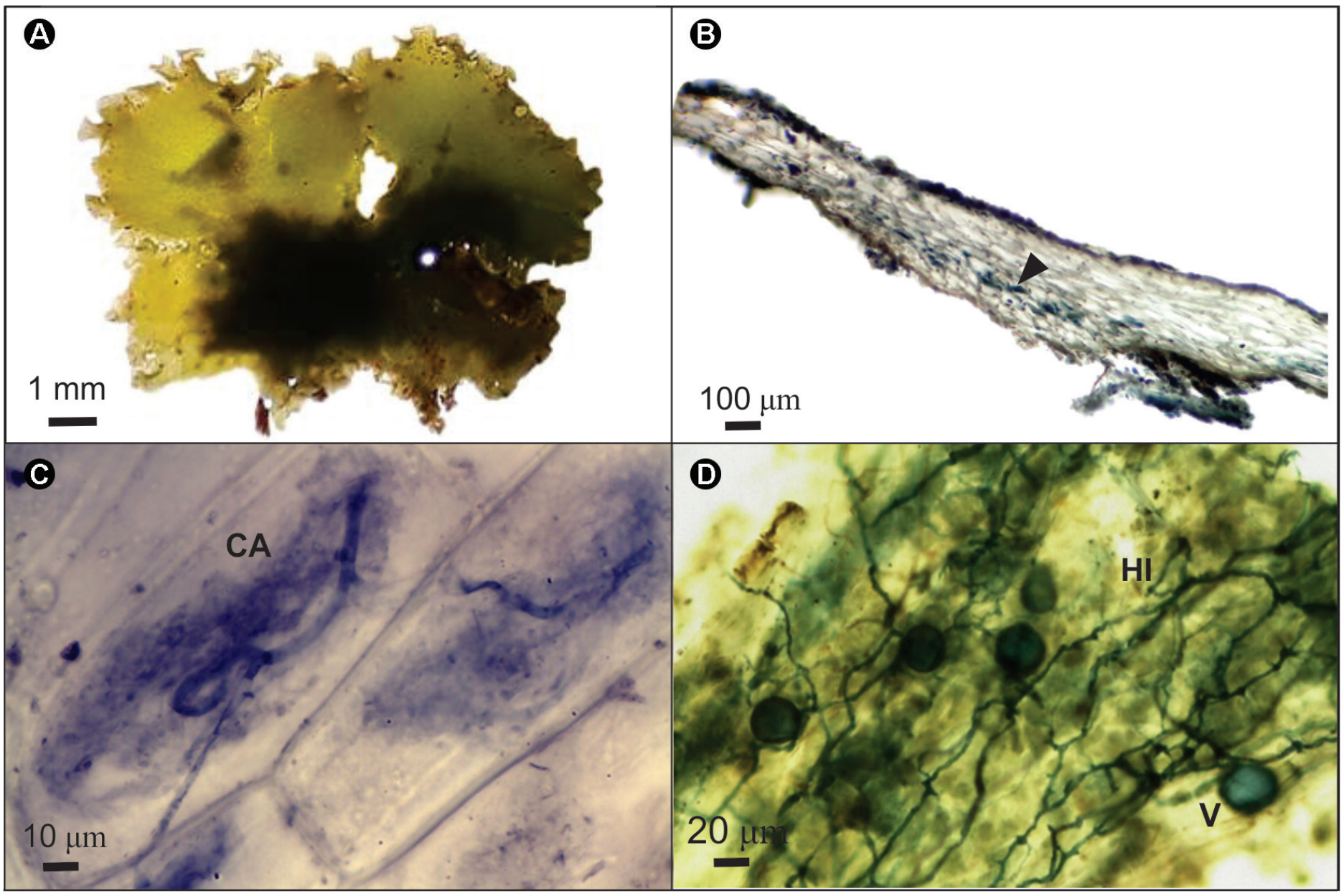

Figura 1: Nothoceros fuegiensis (Steph.) J.C. Villarreal, Cottet 5550 (BCRU). A. aspecto general del talo; B. sección transversal del talo colonizado por hongos micorrícicos arbusculares ( $\nabla$ ); C. coil arbusculado (CA); D. hifas intracelulares (HI) y vesícula (V).

ción, existe el caso de Phaeoceros laevis (L.) Prosk., donde el tipo morfológico de colonización es una forma intermedia entre el tipo Arum y el tipo Paris llamado Intermediate $I$ (Cottet y Messuti, 2017). En los últimos años el estudio de esta simbiosis entre plantas y hongos se ha concentrado en aspectos moleculares y experimentales relacionados con la identidad y fisiología del hongo (Öpik et al., 2010; Martin, 2016; Carrillo-Saucedo y Gavito, 2020). Sin embargo, aún es necesario registrar y describir este tipo de asociación para numerosas especies de briofitas y ambientes. La información brindada a partir de este tipo de estudios descriptivos puede contribuir no solo a la comprensión integral de la interacción que ocurre entre los organismos involucrados, sino también proporcionar nuevos registros sobre la relación entre briofitas y hongos.

\section{Contribución de autores}

ACC coleccionó el material examinado. ACC y MIM contribuyeron a la adquisición de datos, interpretación y escritura, discusión, revisión y aprobación del manuscrito final.

\section{Financiamiento}

Este estudio fue apoyado por la Universidad Nacional del Comahue (B207).

\section{Agradecimientos}

Agradecemos por el apoyo brindado al Consejo Nacional de Investigaciones Científicas y Técnicas, a la Universidad Nacional del Comahue y a la Administración de Parques Nacionales. 


\section{Literatura citada}

Bidartondo, M. I. y J. G. Duckett. 2010. Conservative ecological and evolutionary patterns in liverwort-fungal symbioses. Proceedings of the Royal Society B 277(1680): 485-492. DOI: https://doi.org/10.1098/rspb.2009.1458

Brundrett, M. 2004. Diversity and classification of mycorrhizal associations. Biological Reviews 79(3): 473-495. DOI: https://doi.org/10.1017/S1464793103006316

Carrillo-Saucedo, S. M. y M. E. Gavito. 2020. Resilience of soil aggregation and exocellular enzymatic functions associated with arbuscular mycorrhizal fungal communities along a successional gradient in a tropical dry forest. Mycorrhiza 30: 109-120. DOI: https://doi.org/10.1007/s00572-019-00928-9

Cottet, A. C. y M. I. Messuti. 2017. Identificación del tipo morfológico de micorriza arbuscular en Phaeoceros laevis (Anthocerotophyta). Boletín de la Sociedad Argentina de Botánica 52(2): 291-293. DOI: https://doi. org/10.31055/1851.2372.v52.n2.17444

Cottet, A. C y M. I. Messuti. 2019. New evidence about the interactions between liverworts in the genus Symphyogyna (Pallaviciniaceae) and arbuscular mycorrhizal fungi. Symbiosis 79(2): 117-121. DOI: https://doi.org/10.1007/ s13199-019-00634-2

Cottet, A. C., J. M. Scervino y M. I. Messuti. 2018. An improved staining protocol for the assessment of arbuscular mycorrhizal in bryophytes. Boletín de la Sociedad Argentina de Botánica 53(2): 201-206. DOI: https://doi. org/10.31055/1851.2372.v53.n2.20577

Desirò, A., J. G. Duckett, S. Pressel, J. C. Villarreal y M. I. Bidartondo. 2013. Fungal symbioses in hornworts: a chequered history. Proceedings of the Royal Society B 280(1759): 20130207. DOI: https://doi.org/10.1098/rspb.2013.0207

Dickson, S. 2004. The Arum-Paris continuum of mycorrhizal symbioses. New Phytologist 163(1): 187-200. DOI: https:// doi.org/10.1111/j.1469-8137.2004.01095.x

Feijen, F. A. A., R. A. Vos, J. Nuytinck y V. S. F. T. Merckx. 2018. Evolutionary dynamics of mycorrhizal symbiosis in land plant diversification. Scientific Reports 8(1): 10698. DOI: https://doi.org/10.1038/s41598-018-28920-x

Field, K. J., W. R. Rimington, M. I. Bidartondo, K. Allison, D. J. Beerling, D. D. Cameron, J. G. Duckett, J. R. Leake y S. Pressel. 2016. Functional analysis of liverworts in dual symbiosis with Glomeromycota and Mucoromycotina fungi under a simulated Palaeozoic $\mathrm{CO}_{2}$ decline. International Society for Microbial Ecology Journal 10: 1514-1526. DOI: https://doi. org/10.1038/ismej.2015.204

Frahm, J. P. 2003. Manual of tropical bryology. Tropical Bryology 23(1): 1-200. DOI: https://doi.org/10.11646/bde.23.1.1

Hässel de Menéndez, G. G. 1961. Estudio de las Anthocerotales y Marchantiales de la Argentina. Opera Lilloana 7: 1-297.

Hässel de Menéndez, G. G. 1990. Las especies de Anthoceros y Folioceros (Anthocerotophyta) de América del Norte, Sud y Central; la ornamentación de sus esporas y taxonomía. Candollea 45: 201-220.

Liepina, L. 2012. Occurrence of fungal structures in bryophytes of the boreo-nemoral zone. Environmental and Experimental Biology 10(1): 35-40.

Martin, F. 2016. Molecular Mycorrhizal Symbiosis. Ed. John Wiley \& Sons, Inc. New Jersey, USA. 506 pp. DOI: https://doi. org/10.1002/9781118951446

Nelson, J. y A. J. Shaw. 2019. Exploring the natural microbiome of the model liverwort: fungal endophyte diversity in Marchantia polymorpha L. Symbiosis 78(1): 45-59.

Öpik, M., A. Vanatoa, E. Vanatoa, M. Moora, J. Davison, J. M. Kalwij, U. Reier y M. Zobel. 2010. The online database MaarjAM reveals global and ecosystemic distribution patterns in arbuscular mycorrhizal fungi (Glomeromycota). New Phytologist 188(1): 223-241. DOI: https: //doi. org/10.1111/j.1469-8137.2010.03334.x

Smith, F. A. y S. E. Smith. 1997. Tansley Review Nº 96. Structural diversity in (vesicular)-arbuscular mycorrhizal symbioses. New Phytologist 137(3): 373-388. DOI:https://doi. org/10.1046/j.1469-8137.1997.00848.x

Spatafora, J. W., Y. Chang, G. L. Benny, K. Lazarus, M. E. Smith, M. L. Berbee, G. Bonito, N. Corradi, I. Grigoriev, A. Gryganskyi, T. Y. James, K. O’Donnell, R. W. Roberson, T. N. Taylor, J. Uehling, R. Vilgalys, M. M. White y J. E. Stajich. 2016. A phylum-level phylogenetic classification of zygomycete fungi based on genome-scale data. Mycologia 108(5): 10281046. DOI: https://doi.org/10.3852/16-042

Villarreal, J. C., B. Goffinet, R. J. Duff y D. C. Cargill. 2010. Phylogenetic delineation of Nothoceros and Megaceros (Dendrocerotaceae). The Bryologist 113(Spring 2010): 106113. DOI: https://doi.org/10.1639/0007-2745-113.1.106 